\title{
Quality of Life of Children and Adolescents with Type 1 Diabetes in Kuwait
}

\author{
M. Abdul-Rasoul ${ }^{\mathrm{a}} \quad$ F. AlOtaibi ${ }^{\mathrm{a}} \quad$ A. Abdulla A. Rahme $^{\mathrm{b}} \quad$ F. AlShawaf ${ }^{\mathrm{b}}$ \\ ${ }^{a}$ Department of Pediatrics, Faculty of Medicine, Health Sciences Center, Kuwait University, ${ }^{b}$ Mubarak Hospital, and \\ 'Dasman Diabetes Institute, Kuwait, Kuwait
}

\section{Key Words}

Children · Adolescents · Quality of life · Childhood

psychology · Pediatric Quality of Life Inventory

\section{Abstract}

Objectives: To evaluate the health-related quality of life (HRQoL) of children and adolescents with type 1 diabetes (T1DM) in Kuwait using the Pediatric Quality of Life Inventory (PedsQL) 4.0 Generic Core Scale and PedsQL 3.0 Diabetes Module, and to identify the risk factors associated with unsatisfactory QoL and their effects on metabolic control. Subjects and Methods: A total of 436 patients (2-18 years) with T1DM (>6 months) and 389 healthy controls, with the parents of both groups, completed the Arabic Generic Core Scale. Those with T1DM also completed the Arabic Diabetes Module. Results: The mean total score of the PedsQL Diabetes Module was $70.2 \pm 9.8$ reported by children and $59.9 \pm$ 11.1 reported by parents (higher scores indicate better $\mathrm{QoL}$ ). Young age and long duration of diabetes were associated with poor QoL ( $<0.001)$. Boys had better total scores than girls in most age groups $(70.3 \pm 9.3$ vs. $52.3 \pm 7.2, p<0.001)$; however, girls did better than boys regarding treatment barriers and adherence $(71.3 \pm 7.8$ vs. $68.1 \pm 6.2, p<0.005)$. High- er HbA1c values were associated with lower QoL scores (31.1 \pm 5.1 at $\mathrm{HbA} 1 \mathrm{c}$ of $15 \%$ vs. $82.5 \pm 6.1$ at $\mathrm{HbA} 1 \mathrm{c}$ of $6 \%, \mathrm{p}<$ 0.0001). Conclusion: HRQoL of children and adolescents with T1DM was consistently poorer than controls. Parents consistently reported poorer QoL scores than their children. We recommend that more support should be provided for the care of children with diabetes in Kuwait.

Copyright $\odot 2013$ S. Karger AG, Basel

\section{Introduction}

Health-related quality of life (HRQoL) has increasingly been acknowledged as an essential health outcome measure in pediatric medicine, especially in the last decade. The development and use of pediatric HRQoL measures are important for identifying at-risk children and applying early intervention programs [1].

Type 1 diabetes mellitus (T1DM) is one of the most common chronic diseases, affecting 1 in every 400-600 children and adolescents [2]. Intensive treatment is essential to prevent or delay acute and chronic complications. This involves multiple daily injections of insulin (or insulin infusion), monitoring daily blood glucose, track-

\begin{tabular}{ll}
\hline KARGER & $\begin{array}{l}\text { ( ) 2013 S. Karger AG, Basel } \\
1011-7571 / 13 / 0224-0379 \$ 38.00 / 0 \quad \text { Karger }\end{array}$ \\
$\begin{array}{l}\text { E-Mail karger@karger.com } \\
\text { www.karger.com/mpp }\end{array}$ & $\begin{array}{l}\text { This is an Open Access article licensed under the terms of the } \\
\text { Creative Commons Attribution-NonCommercial 3.0 Un- } \\
\text { ported license (CC BY-NC) (www.karger.com/OA-license), } \\
\text { applicable to the online version of the article only. Distribu- } \\
\text { tion permitted for non-commercial purposes only. }\end{array}$
\end{tabular}

Majedah Abdul-Rasoul

Assistant Prof. of Pediatric Endocrinology

Pediatric Department Faculty of Medicine

Kuwait University, PO Box 24923, Safat, 13110 Kuwait (Kuwait)

E-Mail majdah.a@ hsc.edu.kw 
ing carbohydrate consumption and participating in regular physical activity. Patients and caregivers have to adapt to a new lifestyle while the developing child or adolescent strives to achieve autonomy. Studies have shown that enhancing the QoL and well-being of children with diabetes is as important as metabolic control in preventing secondary morbidity [3]. Therefore, the main aim of modern diabetes care in children and adolescents has changed from a purely medical approach to one aiming toward optimal glycemic control, normal psychological development and maximum QoL [3].

Until recently, all QoL research in patients with diabetes had been conducted mostly in North America, Australia and the UK [4]. No study from the Arabian Gulf region has been reported. Since there is a great difference in the health care delivery system, religion, culture and family dynamics in different societies, there is a need to do QoL studies in different communities.

Therefore, the objectives of this study were to evaluate the QoL of children and adolescents with T1DM in Kuwait and to identify risk factors associated with poor QoL scores and their effects on metabolic control.

\section{Subjects and Methods}

\section{Participants}

A total of 394 children and 472 parents were approached during a clinical visit. Of these, 377 children and adolescents (aged 5-18 years) with T1DM and 436 parents agreed to participate in the study. The patients were recruited from all 6 governorate hospitals of Kuwait and Dasman Diabetes Center. All had diabetes for more than 1 year. Patients older than 12 years of age signed the written informed consent while parents of those younger than 12 years signed for them.

The control group consisted of 389 children and adolescents visiting clinics for vaccines or standard sport checkups or during visits of their siblings. They were matched with the diabetic group by age, sex, socioeconomic status and residence. Children with chronic diseases were excluded.

\section{Demographic and Diabetes-Related Information Form}

The form contained demographic information, including age, gender, nationality, school performance based on grade point average, and information required to calculate socioeconomic status [5]. Diabetes-related information included age at diagnosis, duration of diabetes, mode of treatment and mean $\mathrm{HbAlc}$ during the previous year.

\section{Measures}

The Pediatric Quality of Life Inventory (PedsQL ${ }^{\mathrm{TM}}$ ) 3.0 Diabetes Module, a multi-dimensional, diabetes-specific instrument that assesses children and adolescents (2-18 years), with both child and parent reports, was used to assess HRQoL [6-9]. This 28-item multidimensional instrument consists of 5 scales: (1) diabetes symptoms (11 items), (2) treatment barriers (4 items), (3) treatment adherence ( 7 items), (4) worry (3 items), and (5) communication (3 items) [8].

Children aged 5-18 years provided self-reports, and parents of children aged 2-18 years provided parent proxy-reports. The items for each form were essentially identical. A 5-point scale was used $(0=$ never a problem, $4=$ almost always a problem $)$. Items were reverse-scored and linearly transformed to a 0-100 scale so that higher scores indicated better QoL [4]. Internal consistency reliability of total scores and subscales on the original form was assessed with Cronbach's alpha. A value of 0.7 was set for measures used to detect differences between groups, and $>0.9$ for interpreting individual scores [7].

The 23-item PedsQL 4.0 Generic Core Scale also includes a child self-report and a parent proxy-report. It has 4 subscales: (1) physical function, (2) emotional function, (3) social function and (4) school function. The scoring system is similar to that of the diabetes module [7]. Both instruments were translated into Arabic and showed good internal consistency reliability and constant validity [8].

\section{Procedure}

Interviews were conducted by research assistants with experience in questionnaire application. The child and parent then completed the QoL questionnaire independently. The study protocol was approved by the joint Ethics Research Committee of Kuwait University and the Ministry of Health.

\section{Statistical Analysis}

All statistical analyses were performed using SPSS for Windows, version 17.0 (SPSS Inc., Chicago, Ill., USA). Descriptive statistics are reported as means \pm SEM. The relationship between diabetes-related QoL and the different variables was assessed with Pearson's correlation coefficients. Unpaired t tests were used to compare scores between groups (e.g. diabetics vs. controls, males vs. females). One-way ANOVA was used to compare age groups. A $p$ value of $\leq 0.05$ was considered statistically significant in all analyses.

\section{Results}

The demographic data of the participants including the diabetes-related information are given in table 1 . The mean $( \pm S D)$ age in the diabetic group was $9.1 \pm 3.7$ years, and for the controls $9.7 \pm 3.6$ years, $p>0.5$ ). Cronbach's alpha coefficient for total scores of both patient and parent reports of the Arabic versions of PedsQL 3.0 Diabetes Module and the Generic Core Scale was 0.85, which is considered satisfactory [10]. It was equally high (0.81$0.84)$ for all age groups and subscales $(0.73-0.8)$. Patient and parent reports had an adequate level of concordance, with coefficient values of at least 0.60 (table 2).

Mean $( \pm \mathrm{SD})$ age at the onset of diabetes was $6.1 \pm 4.2$ years and mean duration of disease was $5.37 \pm 2.8$ years. The mean percentage of $\mathrm{HbAlc}$ was $8.0 \pm 1.6$. Of the 436 patients, 61 (14\%) were taking 1-2 injections of insulin 
Table 1. Demographic data for diabetes and control groups

\begin{tabular}{|c|c|c|c|c|}
\hline & \multicolumn{2}{|c|}{ Diabetes group } & \multicolumn{2}{|c|}{ Control group } \\
\hline & $\mathrm{n}$ & $\%$ & $\mathrm{n}$ & $\%$ \\
\hline \multicolumn{5}{|l|}{ Age groups, years } \\
\hline $2-4$ & 59 & 13.5 & 51 & 13.1 \\
\hline $5-7$ & 87 & 20 & 77 & 19.8 \\
\hline $8-12$ & 193 & 44.3 & 170 & 43.7 \\
\hline $13-18$ & 97 & 22.2 & 91 & 23.4 \\
\hline \multicolumn{5}{|l|}{ Gender } \\
\hline Females & 207 & 47.5 & 143 & 49.5 \\
\hline Males & 229 & 52.5 & 146 & 50.5 \\
\hline \multicolumn{5}{|c|}{ Socioeconomic status } \\
\hline Low & 75 & 17.3 & 63 & 16.2 \\
\hline Medium & 191 & 43.8 & 176 & 45.3 \\
\hline High & 170 & 38.9 & 150 & 38.5 \\
\hline \multicolumn{5}{|c|}{ School performance } \\
\hline Poor & 11 & 2.5 & 9 & 2.3 \\
\hline Average & 35 & 8 & 30 & 7.7 \\
\hline Good & 49 & 11.2 & 54 & 13.9 \\
\hline Very good & 235 & 54 & 206 & 53 \\
\hline Excellent & 106 & 24.3 & 90 & 23.1 \\
\hline \multicolumn{5}{|l|}{ Family dynamics } \\
\hline Both parents & 333 & 76.4 & 301 & 77.4 \\
\hline Single parent & 103 & 23.6 & 88 & 22.6 \\
\hline
\end{tabular}

Table 2. Generic and diabetes-specific QoL scores reported by patients and their parents (mean \pm SD)

\begin{tabular}{lllll}
\hline & $\begin{array}{l}\text { Patient } \\
\text { report }\end{array}$ & $\begin{array}{l}\text { Parent } \\
\text { report }\end{array}$ & $\mathrm{t}$ & $\mathrm{p}$ \\
\hline PedsQL GCS & & & & \\
$\quad$ Physical & $75.1 \pm 11.6$ & $70.2 \pm 8.9$ & 4.03 & 0.01 \\
Emotional & $72.7 \pm 10.4$ & $67.4 \pm 9.2$ & 5.09 & 0.001 \\
Social & $82.3 \pm 9.4$ & $76.2 \pm 8.4$ & 5.82 & 0.001 \\
School & $73.5 \pm 10.9$ & $69.4 \pm 9.8$ & 3.57 & 0.01 \\
\cline { 2 - 5 } Total & $75.6 \pm 11.1$ & $70.1 \pm 10.2$ & 4.78 & 0.001 \\
\hline PedsQL DM & & & & \\
Diabetes symptoms & $63.4 \pm 13.1$ & $59.3 \pm 11.3$ & 4.26 & 0.01 \\
Treatment barriers & $70.3 \pm 11.5$ & $66.9 \pm 12.6$ & 5.31 & 0.001 \\
Treatment adherence & $76.6 \pm 10.2$ & $68.4 \pm 12.3$ & 6.35 & 0.001 \\
Worry & $69.9 \pm 11.1$ & $59.8 \pm 10.2$ & 7.51 & 0.0001 \\
Communication & $75.3 \pm 11.3$ & $63.6 \pm 9.4$ & 4.63 & 0.01 \\
Total & $70.2 \pm 9.8$ & $59.9 \pm 11.1$ & 5.26 & 0.001 \\
\hline
\end{tabular}

GCS $=$ Generic Core Scale; DM = Diabetes Module

per day, 298 (68.3\%) were taking 3-4 injections, and 77 $(17.7 \%)$ were using an insulin pump. HbA1c values increased as children grew older; however, the increase was statistically significant only in the adolescent group [7.1 \pm 1.2 (5-7 years) to $8.9 \pm 1.4$ ( $13-18$ years), $\mathrm{p}<0.001]$.

Quality of Life of Diabetic Children and Adolescents in Kuwait

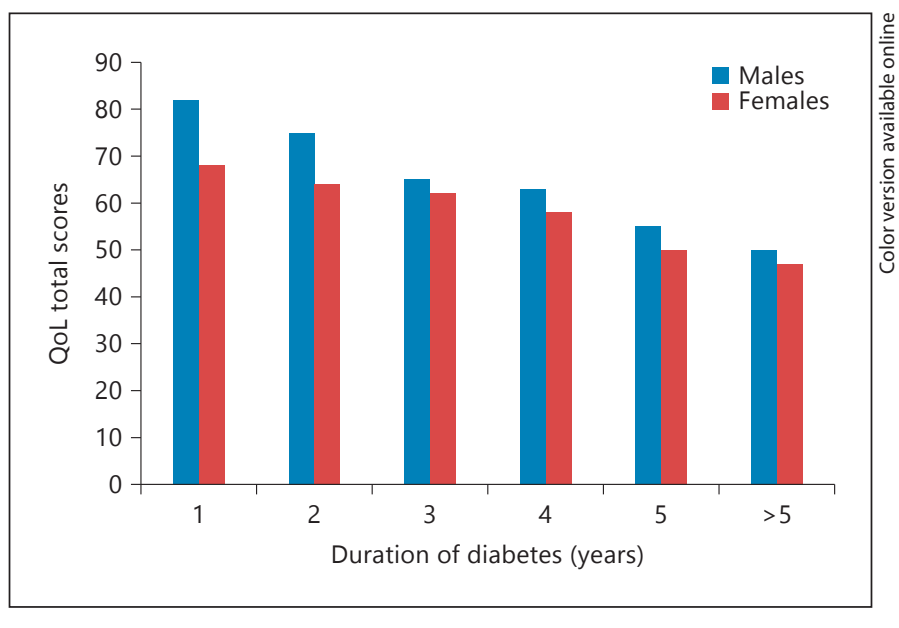

Fig. 1. QoL total scores (Diabetes Module) in relation to duration of diabetes. Total scores for QoL decreased with increased duration of the disease in males and females $(\mathrm{p}<0.5)$.

Children with T1DM reported lower total generic QoL (75.6 \pm 8.9$)$ than controls $(83.5 \pm 9.5, \mathrm{p}=0.001)$. The QoL scores were lower for physical (75.1 \pm 11.6 vs. $84.2 \pm 9.3$, p $<0.05)$ and emotional $(72.7 \pm 10.4$ vs. $84.1 \pm 9.1, \mathrm{p}<0.001)$ domains. There was no difference in the school $(73.5 \pm 9.3$ vs. $73.7 \pm 8.1, \mathrm{p}=0.15)$ and social $(83.2 \pm 8.8$ vs. $83.7 \pm 8.4$, $\mathrm{p}=0.13$ ) QoL scores between patients and controls.

The paired samples $t$ test showed that there was a statistically significant difference between the generic and diabetes-related QoL scores calculated from reports by patients with T1DM compared to scores calculated from the parent reports. The mean total diabetes-related scores from self-reports for children and adolescents was $70.2 \pm$ 9.8, compared to $59.9 \pm 11.1$ based on the reports of their parents $(\mathrm{t}=5.26, \mathrm{p}<0.001)$. The most significant difference was in the worry and treatment adherence scales $(\mathrm{p}<$ 0.0001 and $\mathrm{p}<0.001$, respectively). Regarding generic QoL, the total scores based on reports by patients was 75.6 \pm 11.6 , compared to $70.1 \pm 10.2$ by their parents $(t=4.78$, $\mathrm{p}<0.001)$. Total QoL scores increased with age throughout the age groups $(51,68,58$ and 53 for girls and 52, 62, 62 and 71 for boys, $\mathrm{p}<0.05)$. In most age groups, male sex was associated with better QoL ( $p<0.05$; fig. 1 ). However, when subscale scores were analyzed, girls did better regarding treatment barriers $(70.3 \pm 11.5$ vs. $67.3 \pm 9.8, \mathrm{p}$ $<0.05)$ and treatment adherence $(76.6 \pm 10.2$ vs. $69.7 \pm 8.4$, $\mathrm{p}<0.05)$. Further analysis revealed significant association between QoL score and duration of diabetes $(\mathrm{p}<0.001$; fig. 1).

The use of multiple daily injections was associated with worse QoL in the younger age group $(2-4$ years, $\mathrm{p}<$ 


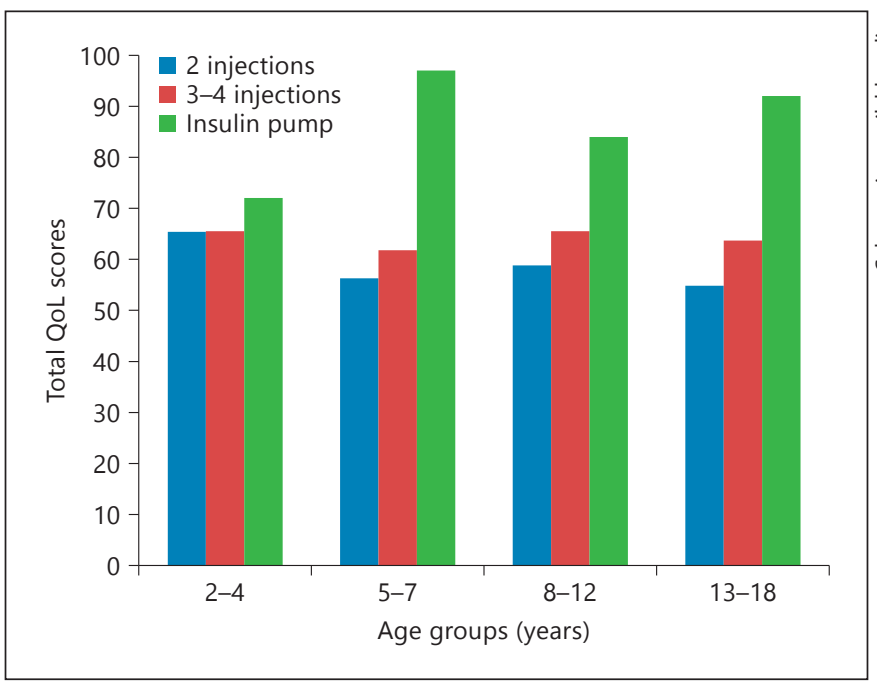

Fig. 2. Total QoL scores (Diabetes Module) in relation to age groups.

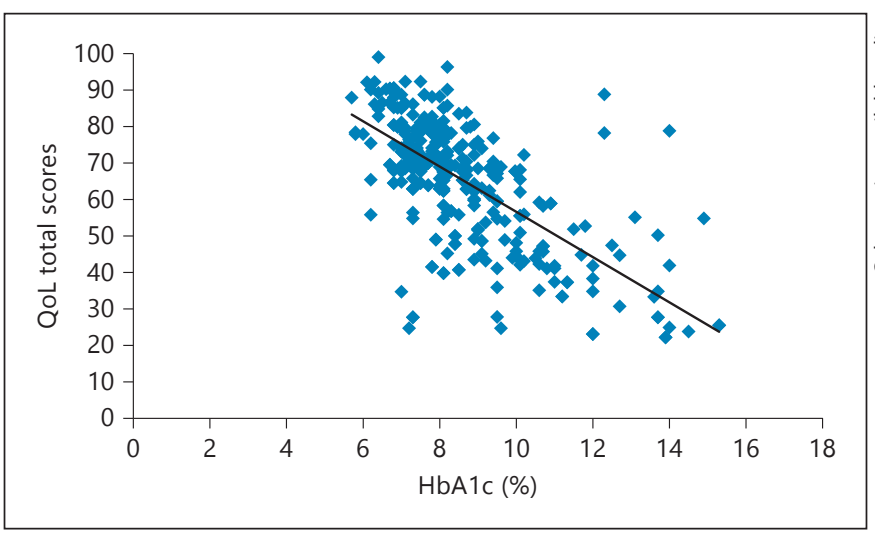

Fig. 3. QoL total scores in relation to HbAlc.

0.05). However, in the other 3 age groups, the use of more than 2 injections was associated with significantly higher scores, according to both child and parent reports $(\mathrm{p}<$ 0.001 ). Moreover, the use of a continuous insulin infusion pump was associated with better QoL in all 4 age groups (fig. 2).

There was a significant correlation between total QoL scores and mean $\mathrm{HbA1c}\left(\mathrm{r}^{2}=-0.7, \mathrm{p}=0.001\right)$. In all age groups, subjects with better metabolic control had better total (fig 3), emotional $\left(\mathrm{r}^{2}=-0.4, \mathrm{p}<0.05\right)$ and social $\left(\mathrm{r}^{2}\right.$ $=0.043, \mathrm{p}<0.05)$ QoL scores.

When generic QoL scores were analyzed against the different variables, HbAlc demonstrated a significant effect on total scores $\left(\mathrm{r}^{2}=-0.44, \mathrm{p}<0.5\right.$; fig 3$)$, as did the duration of diabetes $(\mathrm{p}<0.05)$ and young age at disease onset $(\mathrm{p}<0.01)$. Boys reported better QoL than girls on the generic scale as they did on the diabetes-specific scale $(\mathrm{p}<0.001)$.

\section{Discussion}

The Cronbach alpha coefficient values in this study confirmed the reliability and validity of the Arabic version of the PedsQL 4.0 Generic Core Score and the PedsQL 3.0 Diabetes Module instrument in children with T1DM in Kuwait. Since social and mental well-being is now being acknowledged as an important factor in achieving and maintaining metabolic control and in preventing long-term complications $[9,11]$, there is a need for a reliable tool to assess the effect of diabetes on the QoL of children and adolescents.

Our study has shown that children with T1DM had lower total generic QoL than controls, which is consistent with previous findings of other researchers [10-12]. This is probably due to the demand this disease puts on children and their parents to maintain good glycemic control. QoL scores were low in the emotional and physical domains, and this is also consistent with previous findings $[11,13]$. Lack of autonomy and preoccupation with chronic complications may account for the low emotional QoL $[13,14]$. The children and adolescents in our study did have low scores in school QoL, as was also reported by others $[11,14]$. This was attributed, in 1 report, to frequent absence from school and the subtle neurophysiological changes associated with poor glycemic control [14]. Frequent absence in adolescents was overcome, at least in some places, by having afternoon clinics.

The children and adolescents in the study did not report any problems in social QoL, which other investigators have reported $[11,13]$. Cultural and social factors associated with support from extended family might, at least partly, have helped these children to cope socially within and, subsequently, outside their family. The use of injectable devices (pens, pumps), and more flexible school schedules accommodating diabetes management may help children and adolescents adapt to their condition and socialize with minimal intervention.

Regarding generic and diabetes-related QoL, the discrepancy between patient and parent reports is consistent with previous findings $[10,11,15]$. In all age groups, parents reported poorer QoL than their children and this may indicate the burden of diabetes on the parent, which 
could affect family communication and lead to parental over involvement [16]. In the generic scale, the disagreement between patient and parent reports was most significant in the emotional and social domains. In the diabetes-related QoL, the discrepancy was most significant in the worry and treatment adherence scales. This highlights the importance of not relying only on information collected from caregivers and involving the children and adolescents whenever possible.

Male gender predicted better QoL, as reported in previous studies [3, 10, 14, 17]. Girls have been shown to have more diabetes-related worries and take responsibility for their disease earlier because they tend to enter puberty and mature earlier than boys [15]. Girls were found to be less satisfied with their lives [14]. However, other researchers did not find a relationship between gender and QoL $[18,19]$.

In this study, the age of the patient was an important predictor of QoL in the diabetes module. As children got older, the scores improved; although they changed little in the group aged 13-18 years, they remained significantly higher than in the younger groups, similar to results in many other reports $[10,11,18]$. This could be explained by the fact that adolescents manage their disease more subjectively and independently than younger children [11].

There was a significant relationship between insulin regimen and QoL in all age groups. Patients using insulin pumps, even those less than 4 years old, had better QoL compared to their counterparts taking multiple daily insulin injections as previously reported $[16,17,20]$.

According to the present study, early onset and longer duration of diabetes were associated with poorer QoL, which is consistent with earlier reports [12,21]. In particular, longer duration of diabetes was associated with more psychological and behavioral problems and worse QoL, more so according to parent reports than child and adolescent self-reports [15].

The most important finding of this study is that lower $\mathrm{HbA1c}$ is associated with better QoL, regardless of the duration of the disease. This is similar to results in other reports $[9,10,13,17,20]$. Families of children with good metabolic control reported lower burden and fewer worries than those with poor control. Although a cause-andeffect relation cannot be determined, the association between $\mathrm{HbAlc}$ and QoL is so consistent that it is now justified to consider QoL and metabolic control equally important in the management of T1DM [10-12, 17, 19, 20]. Whether good metabolic control increases QoL or high QoL enhances metabolic control, or both, is an area that requires further study.

The main strength of this study is the high participation rate, which could be partly due to the research not involving any blood sampling nor requiring any extra visits to the hospital.

\section{Conclusions}

The pediatric patients with T1DM and their parents consistently reported lower HRQoL scores compared to healthy controls. Metabolic control, measured by HbA1c value, was significantly associated with QoL in patients with diabetes. There were gender differences, with girls reporting greater difficulties. Perceptions of the family burden by parents were not correlated with patient-rated QoL. We recommend that assessment of QoL after diagnosis of T1DM should be a routine practice in patients with diabetes to facilitate communication, identify early problems and implement early intervention.

\section{References}

$>1$ De Wit M, Delemarre-van de Waal H, Bokma $\mathrm{J}$, et al: Monitoring and discussing health-related quality of life in adolescents with type 1 diabetes improve psychological well-being: a randomized controlled trial. Diabetes Care 2008;31:1521-1526.

$>2$ Roze S, Valentine WJ, Zakrzewska KE, et al: Health-economic comparison of continuous insulin infusion with multiple daily injections for the treatment of type 1 diabetes in the UK. Diabet Med 2005;22:1239-1234.
$>3$ Hoey H, Aanstoot H, Chiarelli F, et al: Good metabolic control is associated with better quality of life in 2,101 adolescents with type 1 diabetes. Diabetes Care 2001;24:1923-1928.

4 Varni J, Burwinkle T, Jacobs J, et al: The Peds$\mathrm{QL}^{\mathrm{TM}}$ in type 1 and type 2 diabetes. Reliability and validity of the Pediatric Quality of Life Inventory ${ }^{\mathrm{TM}}$ Generic Core Scales and type $1 \mathrm{Di}$ abetes Module. Diabetes Care 2003;26:631637.

5 Shah N, Shah M, Radovanovic Z: Towards defining socioeconomic and demographic inequalities that may affect health in Kuwait. Med Princ Pract 1998;7:33-46.
-6 Emmanouilidou E, Galli-Tsinopoulou A, Karavatos A, et al: Quality of life of children and adolescents with diabetes of Northern Greek origin. Hippokratia 2008;12:168-175.

7 Nunnally JC, Bernstein IR: Psychometric Theory. New York, McGraw-Hill, 1994, pp 215-255.

8 Abdul-Rasoul M, AlOtaibi F, AlMahdi M, et al: Reliability and validity of the Arabic version of the PedsQL ${ }^{\mathrm{TM}} 4.0$ Generic Core Score and PedsQL ${ }^{\mathrm{TM}}$ Diabetes Module. Int J Diabetes Mellit 2012;2:301-307. 
9 Jafari P, Forouzandeh E, Bagheri Z, et al: Health-related quality of life of Iranian children with type 1 diabetes: reliability and validity of the Persian version PedsQL Core Scales and Diabetes Module. Health Qual Life Outcome 2011;9:104.

10 Nansel T, Weiseberg-Benchell J, Wysocki T, et al: Quality of life in children with type 1 diabetes: a comparison of general and diabetes-specific measures, and support for a unitary diabetes quality-of-life construct. Diabet Med 2008;25:1316-1323.

11 Kalyva E, Malakonaki E, Eiser C, et al: Healthrelated quality of life (HRQoL) of children and adolescents with type 1 diabetes (T1DM) self and parental perception. Pediatr Diabetes 2011;12:34-40.

12 Graue M, Wentzel-Larsen T, Brue E, et al: The coping styles of adolescents with type 1 diabetes are associated with degree of metabolic control. Diabetes Care 2004;27:1313-1317.
13 Wake M, Hesketh K, Cameron F: The child health questionnaire in children with diabetes: cross-sectional survey of parent and adolescent-reported functional health status. Diabet Med 2000,17:700-707.

14 Al-Akour N, Khader Y, Shatnawi N: Quality of life and associated factors among Jordanian adolescents with type 1 diabetes mellitus. J Diabetes Complications 2010;24:43-47.

15 Nardi L, Zucckini S, D’Alberton F, et al: Quality of life, psychological adjustment and metabolic control in youths with type 1 diabetes: a study with self- and parent-report questionnaires. Pediatr Diabetes 2008;9:496-503.

-16 Hanberger L, Ludvigsson J, Nordfeldt S: Health-related quality of life in intensively treated young patients with type 1 diabetes. Pediatr Diabetes 2009;10:374-381.

17 Wagner VM, Muller-Godeffroy E, von Sengbusch S, et al: Age, metabolic control and type of insulin regimen influence health-related quality of life in children and adolescents with type 1 diabetes. Eur J Pediatr 2005;164:491496.
8 Huang G, Palta M, Allen C, et al: Self-rated health among young people with type 1 diabetes in relation to risk factors in a longitudinal study. Am J Epidemiol 2004;159:364-372.

19 De Wit M, Delemarre-van de Waal H, Bokma J, et al: Self-report and parent-report of physical and psychological well-being in Dutch adolescents with type 1 diabetes in relation to glycemic control. Health Qual Life Outcomes 2007;5:10-18.

20 Vanelli M, Chiarelli F, Chiari G, et al: Relationship between metabolic control and quality of life in adolescents with type 1 diabetes. Report from two Italian centres for the management of diabetes in childhood. Acta Biomed 2003; 74(suppl 1):13-17.

21 Hesketh K, Wake M, Cameron F: Healthrelated quality of life and metabolic control in children with type 1 diabetes: a prospective cohort study. Diabetes Care 2004;27:415-420. 